\title{
О ПРОИЗВЕДЕНИЯХ МОДЕЛЕЙ ДЛЯ ОБОБЩЕННЫХ ЛОГИК
}

(Резюме)

Мы вводим понятие обобшенной логики настолько широкое, что большинство исследуемых в настоящее время логик подходит под это понятие. Логикой мы называем тройку

$$
\mathfrak{i}=\left\langle X, c_{i}^{\mathfrak{x}}, q_{j}^{\mathfrak{x}}\right\rangle_{i \in I, j \in J},
$$

где $X$ - непустое множество, $c_{l}^{\mathfrak{x}}-$ конечно-местные операции, которые мы называем коннективами, $q_{j}^{x}-$ функции из $2^{X}-\{0\}$ в $X$, которые мы будем называть кванторами. При псмощи логических символов соответствующих коннективам и кванторам, предикатов и символов переменных строятся обычным путем формулы данного языка. Модель для логики $\mathfrak{X}$ это пара $\langle\mathfrak{A}, \mathfrak{X}\rangle$ где $\mathfrak{A}=\left\langle A, P_{k}\right\rangle_{k \in K}$, где $A$ - непустое множество, $P_{k}$ - конечно-местные функции из $A$ в $X$. Таким образом обобщаются понятия логического значения, элементарного включения и подмодели. Мы доказываем критерий элементарного включения Тарского-Вота и нижнюю теорему Левенгейма-Сколема.

Для таких логик и моделей кажется натуральным понятие простого произведения такое что

$$
\prod_{r \in R}\left\langle\mathfrak{A}_{r}, \dot{\mathfrak{X}}_{r}\right\rangle=\left\langle\prod_{r \in R} \mathfrak{A}_{r}, \prod_{r \in R} \mathfrak{X}_{r}\right\rangle
$$

где $\prod_{r \in R} \mathfrak{A}_{r}$ и $\prod_{r \in R} \mathfrak{x}_{r}$, вводится обычным путем. При таком определении диагональная модель является элементарной подмоделью простой степени. Другие результаты относятся к обобщенной ограниченной степени и фильтрованному произведению.

Идея такого подхода к логикам и произведениям содержится неявно в статье Фефермана и Вота [3]; см. тоже Войцеховска [8]. 